OPEN ACCESS

Edited by:

Helan Xu,

Jiangnan University, China

Reviewed by: Junheng Zhang,

South-Central University for Nationalities, China Bihe Yuan,

Wuhan University of Technology,

China

*Correspondence:

Zhiming Shi

shizm@imut.edu.cn

Specialty section:

This article was submitted to Polymeric and Composite Materials,

a section of the journal

Frontiers in Materials

Received: 15 May 2021

Accepted: 19 July 2021

Published: 19 August 2021

Citation:

Wang $X$, Shi Z, Zhao $L$ and Shen $X$ (2021) Low Concentration and High

Transparency Keratin Hydrogel

Fabricated via Cryoablation.

Front. Mater. 8:710175.

doi: 10.3389/fmats.2021.710175

\section{Low Concentration and High Transparency Keratin Hydrogel Fabricated via Cryoablation}

\author{
Xiaoqing Wang ${ }^{1,2}$, Zhiming Shi ${ }^{1 *}$, Le Zhao ${ }^{2}$ and Xianyi Shen ${ }^{2}$ \\ ${ }^{1}$ School of Materials Science and Engineering, Inner Mongolia University of Technology, Hohhot, China, ${ }^{2}$ College of Textile and \\ Light Industry, Inner Mongolia University of Technology, Hohhot, China
}

Keratins are highly attractive for medical applications due to their inherent self-assemblies characteristics and biocompatibility. However, nearly all researches have focused on the properties of hybrid hydrogels which was prepared from human hair keratin with other materials, and the preparation methods and properties of pure keratin hydrogels are rarely studied. Thus, we extracted keratins from rabbit hair, and a low concentration and high purity RHK hydrogel was then prepared by a simple freeze-thaw cycle and used to study gelation and the optical properties. The results indicated that RHK keratin hydrogel is a reversible thixotropic system and elastic modulus the storage modulus ( $\left.G^{\prime}\right)$ substantially improves with freeze-thaw cycles. The systematic assessments including microstructural observation, porosity, and the secondary structure confirmed that the structure and properties of keratin hydrogels can be changed by controlling freeze-thaw cycles. Meanwhile, it is found that RHK hydrogel had high optical transmittance, and still maintained its fluorescent properties, which would be useful to observe the wound healing and locate the drug delivery process.

Keywords: keratin hydrogels, freeze-thaw, rheology, morphology, optical properties

\section{INTRODUCTION}

As a renewable resource, keratin resources are very abundant, it is found widely in human and animal organs, including epidermis, hoof, horn, hairs, feather, and protein fiber (Shavandi et al., 2017). For a long time, people have been working on extracting keratin from fiber, studying its biocompatibility and degradability for tissue engineering and medical applications (Esparza et al., 2018a), such as wound healing (Wang et al., 2017; Kim et al., 2019; Konop et al., 2020), hemostatic dressings (Sun et al., 2018; Tang et al., 2021), controlled drug delivery (Guo et al., 2014; Nakata et al., 2015; Sun et al., 2016), and antibacterial wound dressing (Zhai et al., 2018; Sadeghi et al., 2020). Using a riboflavinSPS-hydroquinone (initiator-catalyst-inhibitor) photosensitive solution, Placone and others prepared keratin scaffolds through 3D printing technology (Placone et al., 2017). Despite these advantages, these biomaterials are mainly prepared from keratin mixed with other materials because pure keratin template has poor mechanical properties. The tensile strength and elongation of the keratin-sodium alginate scaffold were intensively studied by Gupta and Hartrianti (Gupta and Nayak 2016; Hartrianti et al., 2017). However, the introduction of other substances may reduce the biocompatibility of the eventual scaffold.

Some studies have tried to make pure keratin scaffolds (Saul et al., 2011; Burnett et al., 2013; Wang et al., 2015), but most of the preparation and research of hydrogels has focused on human hair. However, the color of hair might vary considerably and contain a variety of chemical dye 
composition; in addition, there are high concentration of keratin solution and poor formability of pore. It is well known that the efficiency of extracting keratin is low, which greatly limits the industrial process of preparing hydrogels with keratin. Therefore, it is particularly necessary to find suitable keratin resources and prepare keratin gel with low concentration and high purity by environmentally friendly methods.

As a simple and clean technique for preparing hydrogels, cryogelation has been used more and more. The preparation of keratin hydrogels by cryogelation is mainly based on the selfassembly of keratin. The solvent crystallizes when the temperature is below freezing point, and the keratin macromolecules combine with each other by disulfide bonds, hydrogen bonds, hydrophobicity, electrostatic attraction, and van der Waals forces (Esparza, 2018b), when the temperature returns to above freezing point, as the solvent crystals melt, solvent and other components are enveloped in a three-dimensional network of keratin, an interconnected microporous hydrogel structure is formed (Lozinsky 2002; Henderson et al., 2013). In a recent study by Chua et al. (Cui et al., 2019; Chua et al., 2020; Zhao et al., 2020), HHK sponges was prepared by this method. However, as with previous studies, the main research focuses on the mechanical and biocompatibility of hydrogels, whether pure or hybrid keratin hydrogels.

Herein, keratin extracted from rabbit hair with rich resources, high amino acid content and poor spinnability was used as the research object, a low concentration, high purity, and transparent keratin hydrogel was prepared by simple freeze-thaw (FT) cycle. It has been confirmed that the extracted rabbit hair keratin is nontoxic. In this study, the feasibility of preparation of rabbit hair keratin (RHK) hydrogel by cryogelation was studied. The morphology and structure of RHK hydrogel were tested, and the optical properties of keratin were comprehensively evaluated.

\section{EXPERIMENTAL}

\section{Materials}

Rabbit hairs were collected from German Angora rabbit warren (Gansu, China). Urea and sodium bisulfite were purchased from Damao chemical reagent Co. Ltd (Tianjin, China). Sodium dodecyl sulfate (SDS) was purchased from Usolf Co. Ltd. (Shenzhen, China).

\section{Preparation of Rabbit Hair Keratin}

RHK was extracted according to literature (Wang et al., 2021), with modifications. Briefly, the rabbit hair defatted with petroleum ether and anhydrous ethanol was subjected to ultrasonic treatment. And then, it was immersed in mixed urea-sodium bisulfite-sulfate solvent, and the solution was heated and mechanically stirred for $4.5 \mathrm{~h}$. The solution was filtered to remove the undissolved rabbit hair, and the obtained rabbit keratin solution was subsequently dialyzed in distilled water using a dialysis tube for $48 \mathrm{~h}$ to remove small molecules and salt formed in the reaction; during which, the distilled water was changed every $4 \mathrm{~h}$. The dialysis solution was stored at $4^{\circ} \mathrm{C}$ for the following experiments.

\section{Freeze-Thaw Cycles for Rabbit Hair Keratin Hydrogel}

Dialyzed solution with mass concentration of $2 \%$ was kept in a refrigerated circulating device at $-20^{\circ} \mathrm{C}$ for $12 \mathrm{~h}$, thawed at $4^{\circ} \mathrm{C}$, and then the RHK hydrogel was prepared after several FT cycles. The hydrogel was freeze-dried at $-80^{\circ} \mathrm{C}$ to prepare keratin scaffolds for morphology and structure testing, and the RHK solution without the FT treatment was used as control.

\section{Characterization and Measurements}

Oscillatory rheology: The self-assembly gelation and the change of rheological characteristics during reversible phase transition of keratin hydrogels were characterized using a rheometer (Anton Paar MCR92, Germany). RHK hydrogels were cast in $25 \mathrm{~mm}$ Petri dishes. The oscillatory frequency sweep experiments were performed at angular frequency of $0.1-100 \mathrm{rad} / \mathrm{s}$ at a constant strain of $1 \%$ at $25^{\circ} \mathrm{C}$, and the elastic modulus $\left(\mathrm{G}^{\prime}\right)$ and viscous modulus $\left(G^{\prime \prime}\right)$ were recorded. The apparent viscosity change of $50 \mathrm{~mL}$ keratin solution during gelation was measured using a rheometer (Brookfield R/S Plus Rheometer, United States) at $25^{\circ} \mathrm{C}$.

Scanning electron microscopy (SEM): The RHK scaffold was cut into thin slices with a sharp knife to expose clean cross sections. Samples were gold-sputtered at $18 \mathrm{~mA}$ and observed using a scanning electron microscope (650 FEG, FEI Quanta) at an accelerating voltage of $20 \mathrm{kV}$ under high vacuum. We characterized the pore size within the hydrogel microarchitecture from the SEM images using image processing techniques.

Porosity measurement: The porosity of the RHK scaffold was tested using a liquid displacement method with absolute ethanol (Loh and Choong, 2013). Briefly, put the RHK scaffold into a certain volume $\left(\mathrm{V}_{1}\right)$ of ethanol and record the volume $\left(\mathrm{V}_{2}\right)$ of the solution after $1 \mathrm{~h}$. Next, the liquid-impregnated scaffold was removed, and the remaining liquid volume $\left(\mathrm{V}_{3}\right)$ was recorded. The porosity of the RHK scaffold was calculated according to Equation (1) as follows:

$$
\text { porosity } \%=V_{1}-V_{3} / V_{2}-V_{3} \times 100 \% \text {. }
$$

Fourier transform infrared spectroscopy: Chemical structures of RHK hydrogels and RHK were characterized using a Fourier transform infrared (IR Affinity-1 FTIR, Shimadzu) spectroscope operated in the transmission mode. The FTIR spectra were recorded at a wave number range of $4,000 \mathrm{~cm}^{-1}$ to $400 \mathrm{~cm}^{-1}$, at a resolution of 4.0 , and at 40 scans per sample.

Raman spectroscopy: Raman spectra were obtained via a Raman microscope (INVIA REFLEX03040404, Renishaw). The laser excitation was provided with an argon ion laser operating at $10.2 \mathrm{mw}$ of $633 \mathrm{~nm}$ output. Spectra were recorded between 2,000 and $300 \mathrm{~cm}^{-1}$. For each sample, about three replicates were measured.

X-ray diffraction: Crystal structures of RHK hydrogels and RHK were determined by X-ray diffractometer (D/MAX-2500/ $\mathrm{PC} \mathrm{XRD}$, Rigaku) operated using a $\mathrm{Cu} \mathrm{Ka}$ radiation source. The samples were scanned at a $2 \alpha$ Bragg angle range of $5^{\circ}-60^{\circ}$ at $0.02^{\circ}$ step size and a scan speed of $3 \% \mathrm{~min}$.

Optical properties: The transmittance of RHK samples was measured by UV-visible spectrophotometer (UV-2700, Shimadzu). The scanning range is $300-800 \mathrm{~nm}$. Endogenous 
A

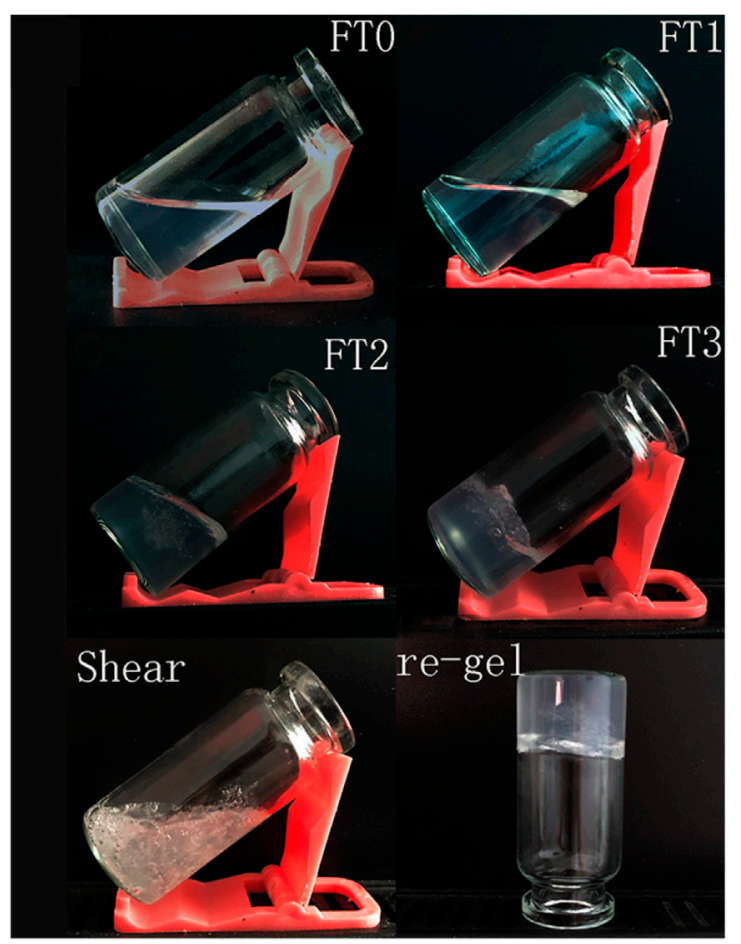

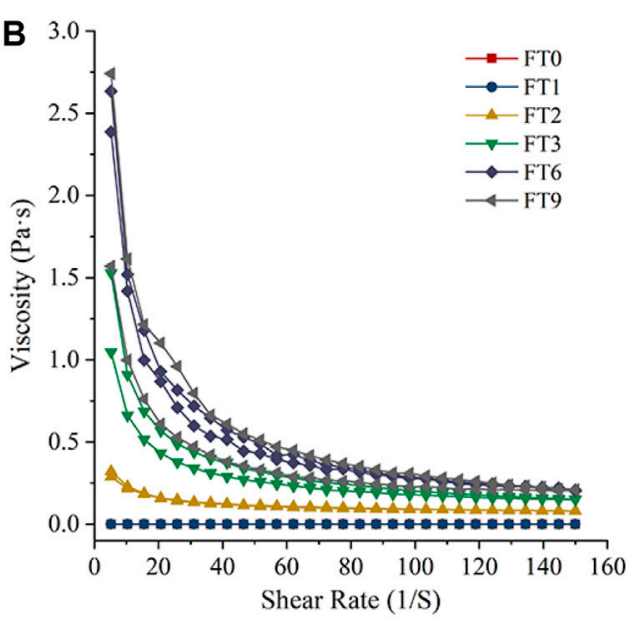

C

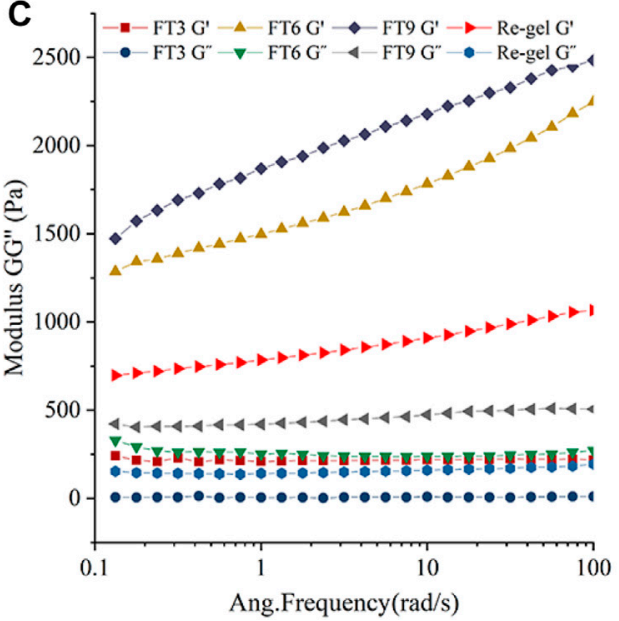

FIGURE 1 | (A) Gelation and Reversible transformation of RHK, (B) viscosity and, (C) oscillatory amplitude sweep measurement of RHK samples at varied FT cycles.

fluorescence spectra of proteins were determined at room temperature using fluorescence spectrophotometer (G9800A, Agilent). The excitation wavelength is $300 \mathrm{~nm}$, and the scanning range is $300-550 \mathrm{~nm}$.

Measurement of particle size distribution: The particles size distribution of RHK butadiene was determined by dynamic laser scattering at $25^{\circ} \mathrm{C}$.

Compressive measurement: Keratin hydrogels were prepared into cylinders with a diameter of $10 \mathrm{~mm}$ and a height of $13 \mathrm{~mm}$. Uniaxial compression was conducted at RT using a universal mechanical tester (Shimadzu, Japan) with $30 \mathrm{kN}$ load cell. The cross-head speed was set at $0.1 \mathrm{~mm} / \mathrm{min}$ and the tests were terminated.

\section{RESULTS AND DISCUSSION}

\section{Rheological Properties of Rabbit Hair Keratin Hydrogels}

The gelation process of low concentration RHK solution was illustrated in Figure 1A. The RHK solution self-assembled into hydrogels without the adding of any chemical cross-linking agent under FT cycles. The transparent and flowing RHK solution was gradually converted to a semi-solid gel state through two FT cycles. With the continuation of FT, RHK solution forms a single highly transparent, intact piece of gel with a three-dimensional network structure. The RHK hydrogel (FT3)broken by shearing can form the initial gel state after being cultured at $-20^{\circ} \mathrm{C}$ for $12 \mathrm{~h}$ (Re-gel). From this knowable, RHK hydrogel is a reversible thixotropic system. Noticeable feature is the absence of distinct phase separation between the keratin hydrogels and the surrounding aqueous medium as the article says (Zhao et al., 2020). We believe that the formation of keratin hydrogels is due to the three-dimensional network structure formed by the binding of keratin molecules, bound water adsorbed by keratin hydrophilic groups, and the unbound water clamped in the pore structure.

In order to further elucidate the rheological properties of lowconcentration RHK solution during FT cycles, the apparent viscosity, $G^{\prime}$, and $G^{\prime \prime}$ measurements were performed on the RHK samples. In Figure 1B, comparative analysis between RHK samples after different FT cycles shows that the apparent 

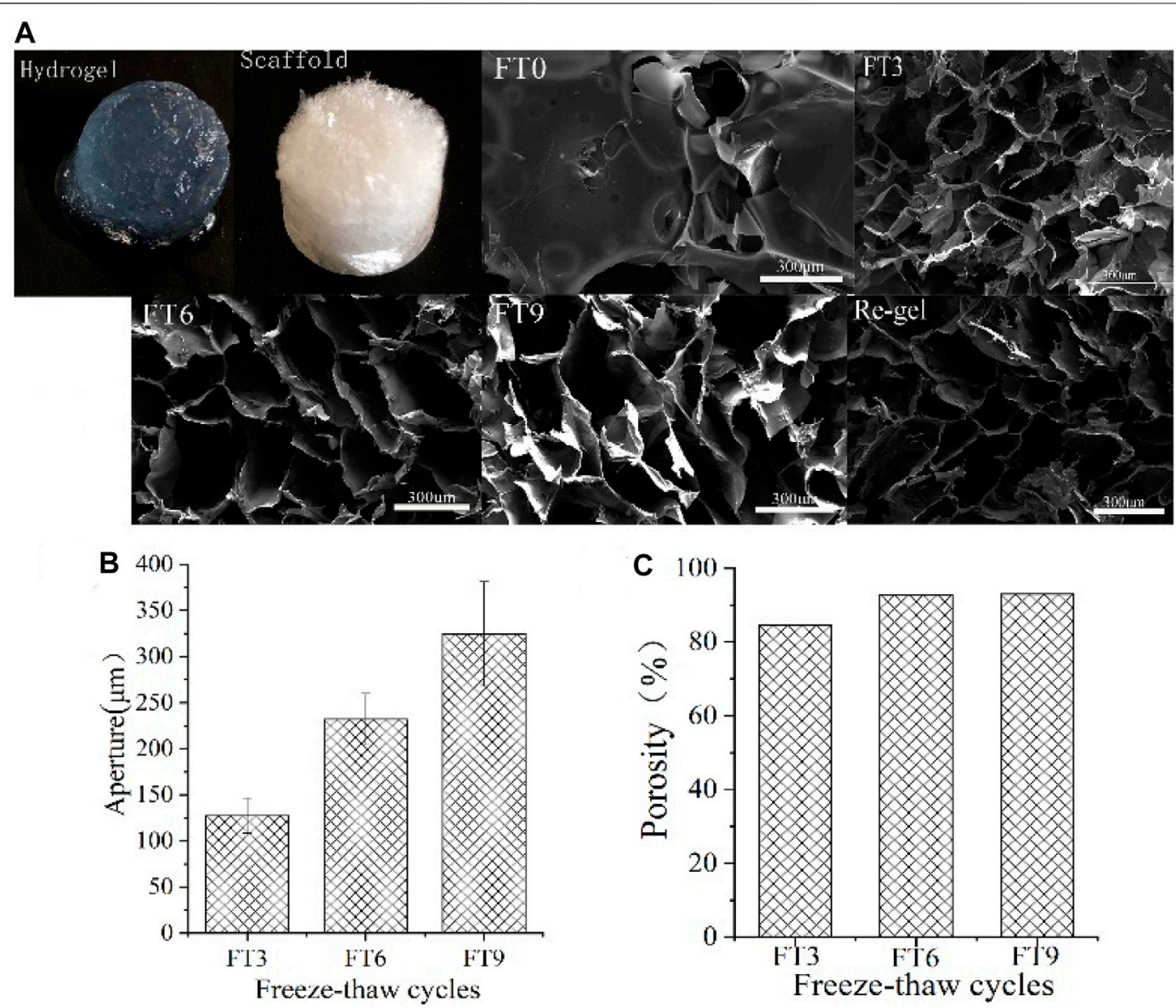

FIGURE 2 | (A) SEM images, (B) pore size distribution, and (C) porosity.

viscosity of RHK solution did not change and was almost zero, this is consistent with the actual gelation phenomenon (Figure 1A-FT1) after one FT cycle. The apparent viscosity of the RHK hydrogels increased with the number of FT cycles after FT2. For all samples, the apparent viscosity decreased with increasing shear rates, suggesting a shear-thinning behavior that is typical of viscoelastic fluids and gels, which could be attributed of weak interactive forces or chain entanglements between RHK at high shear rates.

$\mathrm{G}^{\prime}$ is used to evaluate the strength or solid-like behavior of hydrogels. It is related to the ability of material to store energy and return to its original shape after being subjected to stress. Assessment on the viscoelastic properties of hydrogels (FT3, FT6, and FT9) and re-gel sample was conducted with oscillatory rheology (Figure 1C). The experiments show that the $G^{\prime}$ is significantly higher than $G^{\prime \prime}$, thereby suggesting that these samples behave more like a viscoelastic gel rather than a viscous fluid (Fu et al., 2020). Furthermore, $\mathrm{G}^{\prime}$ increased with the FT cycles, with the highest recorded value of 2,483.3 $\mathrm{Pa}$ in FT9 samples, which indicated that the rheological properties of the hydrogels depended on the FT cycles. It is interesting that the $\mathrm{G}^{\prime}$ and $G^{\prime \prime}$ of re-gel sample were higher than that of FT3 samples, and it can be seen that the shear force cannot cause irreversible rupture of the keratin hydrogels.

\section{Rabbit Hair Keratin Scaffolds Morphology}

Bioporous materials not only provide space for cell proliferation and survival (Wang et al., 2012) but also provide a microenvironment for the maintenance and release of bioactive molecules (Chen and Mooney 2003). To further elucidate the correlation between macroscopic behavior and micro morphology, the morphologies of RHK scaffolds freeze-dried at $-80^{\circ} \mathrm{C}$ with different FT cycle were shown in both low- and highmagnification SEM images (Figure 2A). The morphologies of RHK solution sample freeze-dried at $-80^{\circ} \mathrm{C}$ presented obvious lamellae albeit structure, but the morphology of RHK scaffolds appears to have regions of regular porous structure with interconnected pores. Cui et al. (2019) prepared the hydrogel with $20 \mathrm{mg} / \mathrm{ml}$ pure human hair keratin solution, which is the relatively low concentration used for preparation of keratin hydrogel at present. However, the microstructural imaging shows that the porous structure of keratin hydrogel is incomplete.

Ge et al. (2021) prepared rabbit hair keratin with L-cysteine hydrogel by the heating and cooling method, and the keratin 

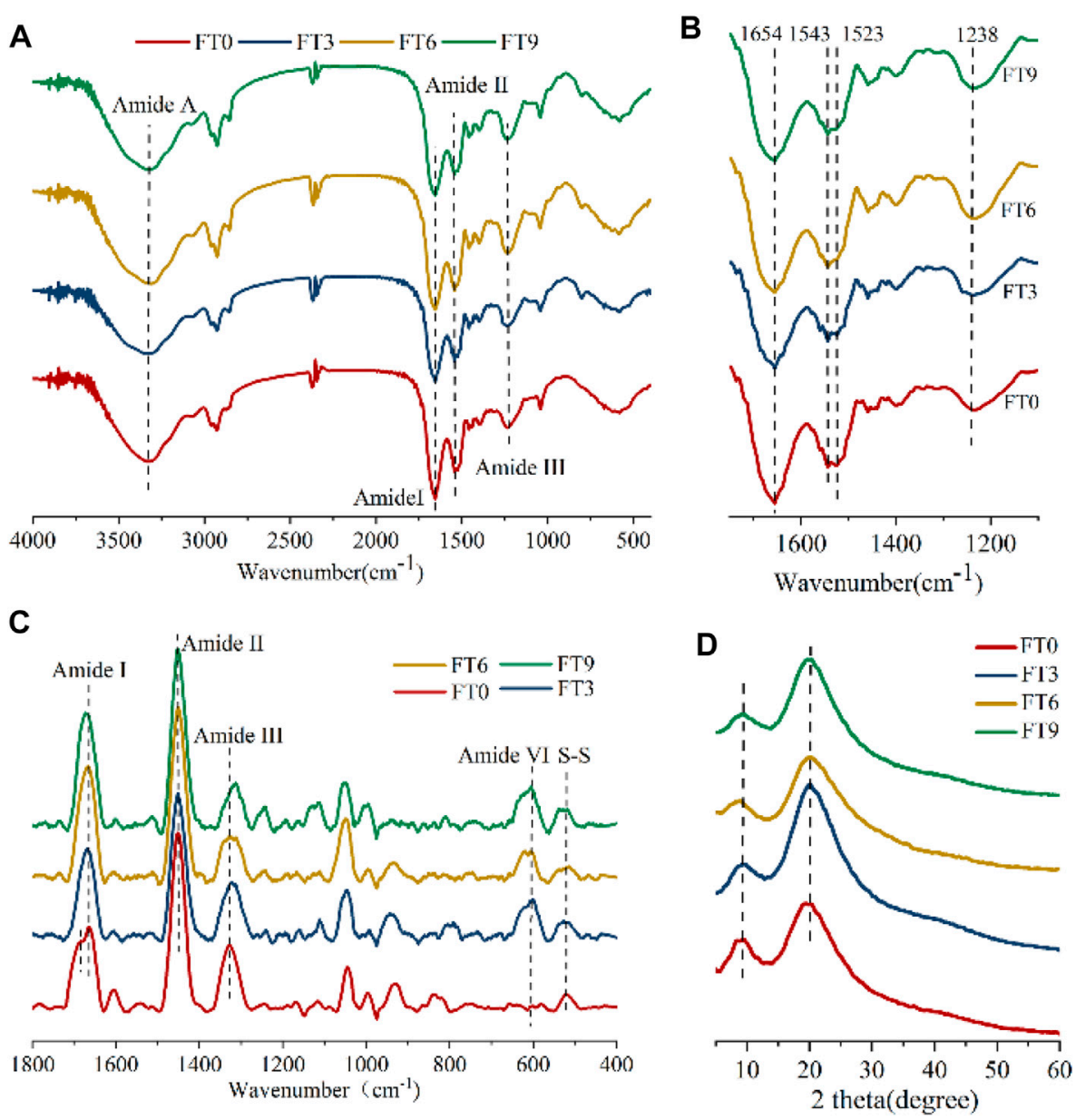

FIGURE 3 | (A-B) The FTIR spectra of RHK samples, (C) Roman spectra, and (D) XRD.

hydrogel showed an uneven porous structure. The shape of the hole gradually changes from approximately round to rectangular as FT cycles increases, and even to flake. At the same time, the wall thickness of the hole also increases gradually. This distinction in morphology is evident in FT3 and FT9 samples. It can be inferred that the formation of columnar ice crystals affects the shape of keratin hydrogel pores during FT processes. The damaged hydrogels are still able to maintain intact porous structures after the FT cycle.

The maximum distance of each hole was selected for the test because the shape of hole of the FT9 scaffolds tends to be rectangular. Figure 2B shows that the aperture of the threedimensional keratin scaffold increases with the FT cycles. Apertures of the keratin scaffolds were $127 \mathrm{um}, 232 \mathrm{um}$, and 324 um, respectively. Figure 2C shows that porosity of the keratin scaffold is as high as $92 \%$. In addition, FT cycles have a significant effect on aperture, but had no obvious effects on the porosity of keratin scaffolds. After vacuum freeze drying, the moisture content directly affects the porosity because the keratin hydrogels gradually dehydrate to form a sponge scaffold. As can be seen from Figure 1, there is almost no water loss in the process of FT cycles, so the volume of hydrogels had little change after forming, which may be the reason why the FT cycles have little influence on the porosity.

\section{Structure of RHK Hydrogels}

The FTIR spectra of RHK samples were observed in the range of $400-4,000 \mathrm{~cm}^{-1}$ (Figure 3A), where they could be assigned to the stretching vibrations of $-\mathrm{N}-\mathrm{H}$, which occurs in the range of $3,200-500 \mathrm{~cm}^{-1}$ (Amide $\mathrm{A}$ ), the stretching vibration $\mathrm{C}=\mathrm{O}$ which fall at $1,654 \mathrm{~cm}^{-1}$ (Amide I), the out-plane bending vibration of $\mathrm{N}-\mathrm{H}$ and $\mathrm{C}-\mathrm{H}$ stretching which falls in $1,480-1,580 \mathrm{~cm}^{-1}$ (Amide $\mathrm{II})$, and the in-phase stretching vibration of $\mathrm{C}-\mathrm{N}$ which fall at $1,238 \mathrm{~cm}^{-1}$ (Amide III) (Zhang, Zhao, and Yang 2015). Functional groups and chemical bonds were generated after the FT treatment of keratin solution. The amide I region of the infrared spectrum of keratin is shown in Figure 3B. It can be seen that the typical absorption peak at amide I region $1,654 \mathrm{~cm}^{-1}$ of the infrared spectrum of keratin corresponds to the intermolecular $\beta$ folding structure. After FT cycle, the position of the absorption peak did not have any deviation. Compared with amide I, amide II band is mainly sensitive to environmental changes of the $\mathrm{N}-\mathrm{H}$ group. Therefore, the amide II band can be used to infer changes in the hydrogen bond microenvironment. 

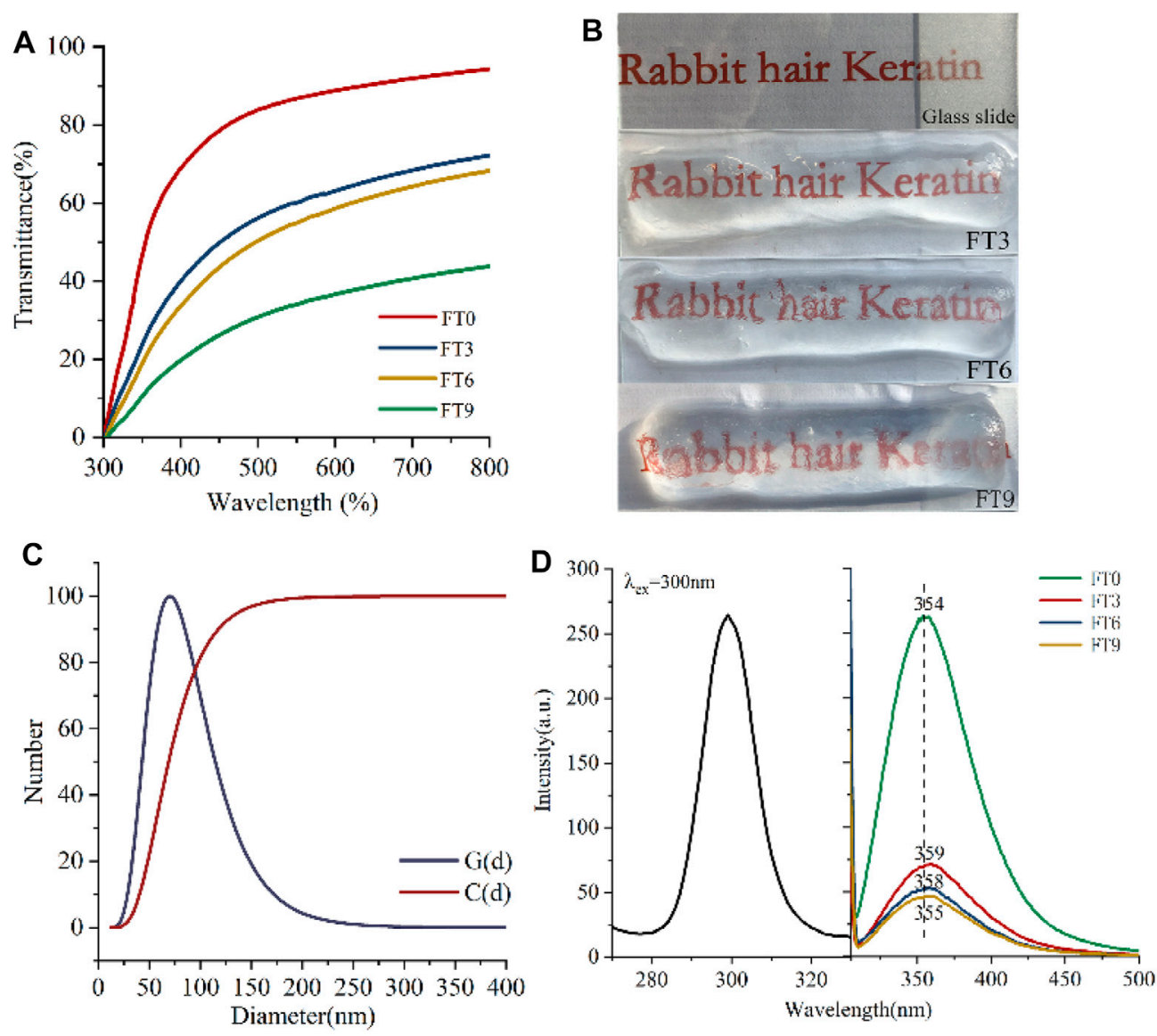

FIGURE 4 | (A) Optical transmittances of keratin hydrogel at varied FT cycles, (B) photographs of the glass and keratin hydrogel, (C) DLS of keratin solution, and (D) room temperature excitation and emission spectra.

Belton studies have found that strong $\mathrm{N}-\mathrm{H}$ hydrogen bond groups are absorbed at high frequencies, that is, the stronger the high wave number absorption, the more the hydrogen bond groups are (Belton et al., 1995; Almutawah et al., 2007). As can be seen from Figure 3B, compared with RHK solution, the absorption peak of RHK hydrogels at $1,543 \mathrm{~cm}^{-1}$ was significantly higher than that at $1,523 \mathrm{~cm}^{-1}$, indicating that hydrogen bond association in macromolecular peptide of keratin was enhanced to varying degrees after FT. This is due to the re-formation of hydrogen bonds between keratin molecules during FT cycles which weakens the association between protein molecules and water molecules.

The Raman spectra of RHK samples were observed in the range of $400-1,800 \mathrm{~cm}^{-1}$ (Figure 3C), where they could be assigned to Amide I $\left(1,597-1,680 \mathrm{~cm}^{-1}\right)$, Amide III $\left(1,229-1,310 \mathrm{~cm}^{-1}\right)$, and the S-S bond $\left(506-550 \mathrm{~cm}^{-1}\right.$ ) (Yu 2002); among them, Amide I and Amide III are very sensitive to protein changes. The absorption peak of keratin solution at $1,684 \mathrm{~cm}^{-1}$ corresponds to the intermolecular $\gamma$ folding structure, which is not present in keratin hydrogels. The peaks of the Amide III are shifted, but all of them are within the range of $1,313-1,337 \mathrm{~cm}^{-1}$, which is a signal of $\beta$-rotation. Compared with keratin solution, the peak of disulfide bond of RHK hydrogel is wider, which may be related to the sulfhydryl group being oxidized to disulfide bond in the transaction process. Notable phenomenon is that the absorption peak of amide $\mathrm{VI}$ region at $604 \mathrm{~cm}^{-1}$ corresponds to the $\mathrm{C}=\mathrm{O}$ outside curved surface signal becomes apparent in the RHK hydrogels.

In the XRD pattern in Figure 3D, the $\alpha$-helix of RHK hydrogels $\left(2 \theta=9.48^{\circ}\right)$ shifted to the lower value compared with RHK solution $\left(2 \theta=19.44^{\circ}\right)$, especially in the FT6 and FT9 samples. At the same time, the $\beta$-sheet of RHK hydrogels $\left(2 \theta=20.06^{\circ}\right)$ shifted to the higher value compared with RHK solution $\left(2 \theta=19.44^{\circ}\right)$. However, the variation of $\beta$-sheet of FT6 and FT9 samples was not as obvious as that of the FT3 sample. This is perhaps because of the increase in the interactions between keratin after several FT cycles. The interactions hindered the $\alpha$-helix to $\beta$-sheet conversion to some extent, while they provided more chance for clustered random coils to form $\beta$-sheet.

\section{Optical Properties of RHK Hydrogels}

Figure 4A shows the result of light transmittance measurements versus wavelength for the keratin before and after the gel. The purified RHK solution offered high transmittance about $88 \%$ in visible wavelengths, and the maximum transmittance of keratin hydrogels prepared by FT can reach $67.57 \%$. The transmittance 
test showed that the transparency of RHK decreased with the increase in FT cycles. Furthermore, the optical transparency of the RHK hydrogels was examined by comparing the appearance of these hydrogels with that of glass substrate (Figure 4B). As shown in the figure, the appearance of hydrogels formed after three FT cycles is similar to that of glass, and the background handwriting can still be seen through hydrogels after nine FT cycles. This phenomenon is rarely reported in other keratin hydrogels.

Pure wool keratin hydrogels concentration of $80 \mathrm{mg} / \mathrm{ml}$ prepared by al.(Chen et al., 2021) at room temperature showed milky white, while pure human hair keratin hydrogels of $20 \mathrm{mg} / \mathrm{ml}$ prepared by Cui et al.(Cui et al., 2019) at $-20^{\circ} \mathrm{C}$ showed yellowish white. Keratin hydrogels are used as medical adjuvant, and its high transparency is conducive to observing the healing of wounds.

The high transparency of RHK hydrogels is not only related to the low concentration of $2 \%$ keratin but also related to the degree of cross-linking of macromolecular chains and the uniformity of pore size distribution in the process of gel formation (Chen 2002). During the FT process, the three-dimensional network structure of RHK hydrogels is formed through the cross-linking of keratin macromolecules, and there is liquid water, uncross-linked keratin molecules, and cross-linked regions in the hydrogels. The existence of liquid water would not affect the transparency, and the uncross-linked keratin molecules will have a certain influence on the transparency of the hydrogel (Hassan and Peppas 2000). Therefore, the particle size of keratin was measured as shown in Figure 4C. RHK particle size distribution curve $[G(d)]$ results show that the particle size of keratin mainly concentrates between 25 and $-150 \mathrm{~nm}$, and the particle size accumulation curve $[\mathrm{C}(\mathrm{d})]$ data show that the number percentage of particle size less than $100 \mathrm{~nm}$ is more than $82.5 \%$. The particle size was less than half of the incident light, and the substance formed was relatively transparent. It can be seen that the small particle size and narrow distribution range of keratin may also be one of the reasons for the formation of transparent hydrogels. The transmittance of RHK hydrogels decreases with the increase in FT cycles, which may be due to the high crystallinity and surface roughness caused by the aggregation and cross-linking of keratin molecules. At the same time, SEM results show that the pore size is greatly different, and the distribution of keratin hydrogels is uneven with the increase in FT times, which will cause serious light scattering and thus affect the transparency.

Fluorescent biomaterials have always received attention because they can be tracked in vivo and needs no external fluorophore. Keratin is a biological material with natural fluorescent properties because it contains residues of tryptophan, tyrosine, and phenylalanine, which absorb ultraviolet light and emit fluorescence. To study whether it still had the inherent protein fluorescence properties, we examined the emission spectra of the keratin hydrogels. Figure 4D shows the emission spectra of RHK solution and hydrogels. When monitored at $354 \mathrm{~nm}$, the obtained emission spectrum of keratin solution consisted of one broad band in the wavelength range of $280-320 \mathrm{~nm}$ with a peak at $300 \mathrm{~nm}$. The resulting emission spectrum was composed of a broad emission band in the wavelength range from 300 to $500 \mathrm{~nm}$, and emission peak was found to be $354 \mathrm{~nm}$, which was close to fluorescence spectra of tryptophan (Burstein et al., 2001). Besides, the stoke shift and full width at half maxima (FWHM) of the emission band were calculated as 54 and $67 \mathrm{~nm}$, respectively.

The inherent fluorescence wavelength and fluorescence intensity of protein are affected by the tertiary structure of protein, the spatial distribution of fluorescent amino acids in protein, the interaction of side chain groups with other residues or solvents, and the energy resonance transfer within fluorescent amino acids (Ross et al., 1997). Compared with RHK solution, the peak generates bathochromic shift in the emission spectrum of hydrogels and the fluorescence intensity is reduced. This may be because disulfide bond, hydrogen bond, ionic bond, and hydrophobic bond are formed in the gelation process of keratin solution, which strengthens the interaction between proteins and reduces the amount of charge on the surface of protein molecules, leading to the gradual exposure of the side chain groups of fluorescent amino acid molecules to the aqueous solution. At this time, the polarity of the environment in which the chromogenic amino acid is located gradually increases, the extension degree of the peptide chain increases, and the content of helical structure decreases. This was also confirmed by the structural test results of keratin hydrogels.

The transmittance test showed that the transparency of keratin hydrogels decreased with the increase in FT cycles. The fluorescent biomaterial test results also showed that the more the FT cycles of keratin hydrogels, the smaller the red shift

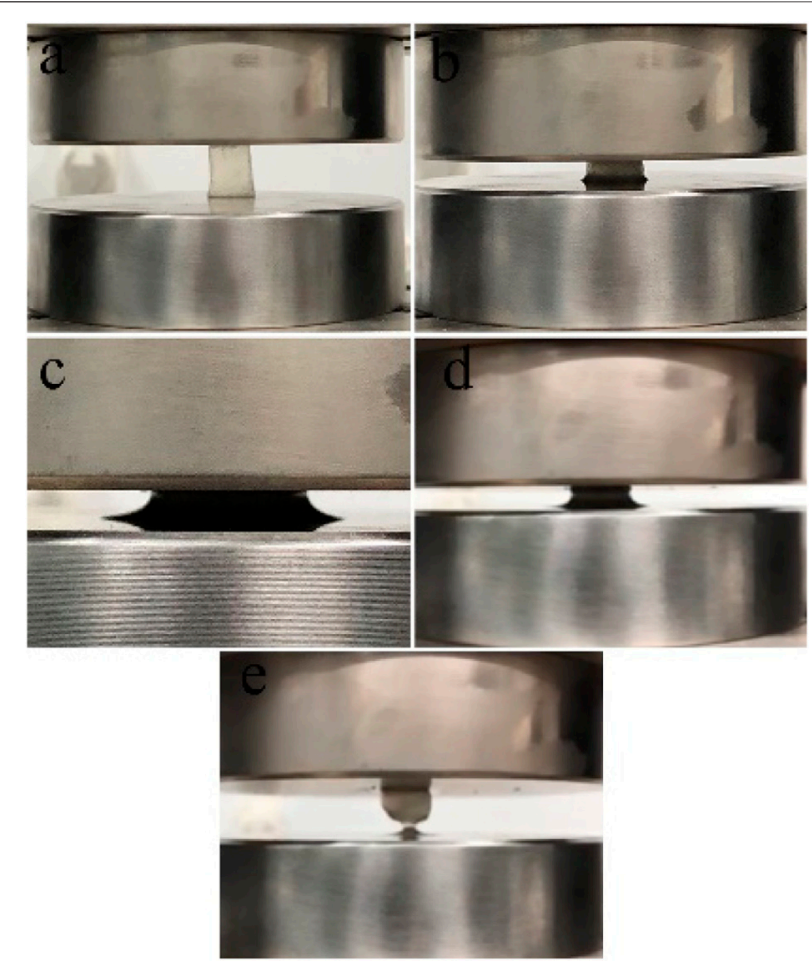

FIGURE 5 | Compression process of RHK scaffolds. 


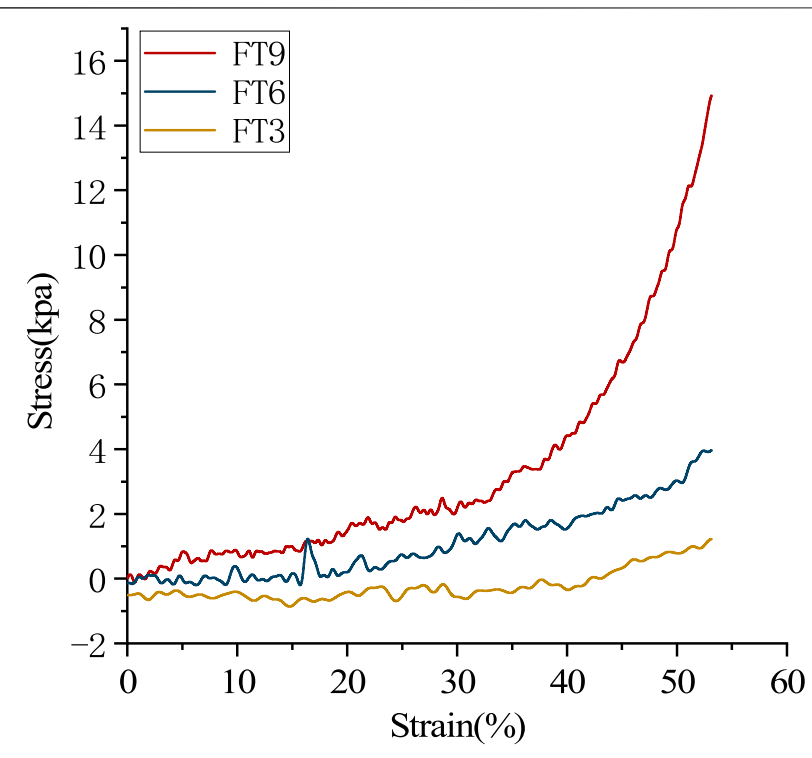

FIGURE 6 | Stress-strain curve.

of the emission spectrum peak and the lower the fluorescence intensity. This is also related to the fact that a small amount of water will be removed from the keratin hydrogels after multiple FT cycles.

\section{Compressive Properties}

The compressive properties of the RHK hydrogel are presented in Figure 5 and Figure 6. From Figure 5, the deformation of keratin hydrogel increases continuously under the action of pressure, the hydrogel did not break after the removal of external force when the deformation reached more than $80 \%$, and the deformation of the hydrogel recovers. The reduced volume of keratin hydrogel after deformation recovery is due to the removal of partial unbound water under external force. It can be seen that the keratin hydrogel has good elasticity. The compressive strength

\section{REFERENCES}

Almutawah, A., Barker, S. A., and Belton, P. S. (2007). Hydration of Gluten: a Dielectric, Calorimetric, and Fourier Transform Infrared Study. Biomacromolecules 8, 1601-1606. doi:10.1021/bm061206g

Belton, P. S., Colquhoun, I. J., Grant, A., Wellner, N., Field, J. M., Shewry, P. R., et al. (1995). FTIR and NMR Studies on the Hydration of a High-Mr Subunit of Glutenin. Int. J. Biol. Macromolecules 17, 74-80. doi:10.1016/0141-8130(95) 93520-8

Burnett, L. R., Rahmany, M. B., Richter, J. R., Aboushwareb, T. A., Eberli, D., Ward, C. L., et al. (2013). Hemostatic Properties and the Role of Cell Receptor Recognition in Human Hair Keratin Protein Hydrogels. Biomaterials 34, 2632-2640. doi:10.1016/j.biomaterials.2012.12.022

Burstein, E. A., Abornev, S. M., and Reshetnyak, Y. K. (2001). Decomposition of Protein Tryptophan Fluorescence Spectra into Log-Normal Components. I. Decomposition Algorithms. Biophysical J. 81, 1699-1709. doi:10.1016/s00063495(01)75823-7

Chen, F. S. (2002). Studies on Optical Properties and Applications of Soybean Protein Gels. beijing: China agricultural university. was also significantly increased with more freeze-thaw cycles (Figure 6). The compressive stress of RHK hydrogel (FT9) reached to $14.927 \mathrm{kPa}$ when the compressive strain is $53 \%$.

\section{CONCLUSION}

In a nutshell, low concentration and highly pure RHK hydrogels were fabricated by green and simple freeze-thaw technique without adding extraneous reagents. The morphology, structure, and physical properties of RHK hydrogels can be adjusted by controlling the FT processing parameters. The RHK hydrogels shows unique optical properties, and its transparency is up to $67.57 \%$, while maintaining its fluorescence properties, which is conducive to the observation of wound healing and location of drug delivery.

\section{DATA AVAILABILITY STATEMENT}

The original contributions presented in the study are included in the article/supplementary material, further inquiries can be directed to the corresponding author.

\section{AUTHOR CONTRIBUTIONS}

XW: data curation and writing-original draft. ZS: methodology, supervision, review and editing. LZ and XS: investigation; All authors contributed to the article and approved the submitted version.

\section{FUNDING}

This research was supported by Natural Science Foundation of Inner Mongolia (2020LH05005), and the Foundation of Inner Mongolia University of Technology (ZZ201817).

Chen, M., Ren, X., Dong, L., Li, X., and Cheng, H. (2021). Preparation of Dynamic Covalently Crosslinking Keratin Hydrogels Based on Thiol/disulfide Bonds Exchange Strategy. Int. J. Biol. Macromolecules 182, 1259-1267. doi:10.1016/ j.ijbiomac.2021.05.057

Chen, R. R., and Mooney, D. J. (2003). Polymeric Growth Factor Delivery Strategies for Tissue Engineering. Pharm. Res. 20, 1103-1112. doi:10.1023/a: 1025034925152

Chua, H. M., Zhao, Z., and Ng, K. W. (2020). Cryogelation of Human Hair Keratins. Macromol. Rapid Commun. 41, 2000254. doi:10.1002/ marc.202000254

Cui, X., Xu, S., Su, W., Sun, Z., Yi, Z., Ma, X., et al. (2019). Freeze-thaw Cycles for Biocompatible, Mechanically Robust Scaffolds of Human Hair Keratins. J. Biomed. Mater. Res. 107, 1452-1461. doi:10.1002/jbm.b.34237

Esparza, Y., Bandara, N., Ullah, A., and Wu, J. (2018a). Hydrogels from Feather Keratin Show Higher Viscoelastic Properties and Cell Proliferation Than Those from Hair and Wool Keratins. Mater. Sci. Eng. C 90, 446-453. doi:10.1016/ j.msec.2018.04.067

Esparza, Y., Ullah, A., and Wu, J. (2018b). Molecular Mechanism and Characterization of Self-Assembly of Feather Keratin Gelation. Int. J. Biol. Macromolecules 107, 290-296. doi:10.1016/j.ijbiomac.2017.08.168 
Fu, Y., Ren, P., Wang, F., Liang, M., Hu, W., Zhou, N., et al. (2020). Mussel-inspired Hybrid Network Hydrogel for Continuous Adhesion in Water. J. Mater. Chem. B 8, 2148-2154. doi:10.1039/c9tb02863c

Ge, N., Zhang, Y., Zhang, H., Zhu, R., and Shi, X. (2021). Preparation and Characterization of Rabbit Hair Keratin Hydrogel. IOP Conf. Ser. Mater. Sci. Eng. 1040, 012003. doi:10.1088/1757-899X/1040/1/012003

Guo, J., Pan, S., Yin, X., He, Y.-F., Li, T., and Wang, R.-M. (2014). pH-Sensitive Keratin-Based Polymer Hydrogel and its Controllable Drug-Release Behavior. J. Appl. Polym. Sci. 132, a-n. doi:10.1002/app.41572

Gupta, P., and Nayak, K. K. (2016). Optimization of Keratin/alginate Scaffold Using RSM and its Characterization for Tissue Engineering. Int. J. Biol. Macromolecules 85, 141-149. doi:10.1016/j.ijbiomac.2015.12.010

Hartrianti, P., Nguyen, L. T. H., Johanes, J., Chou, S. M., Zhu, P., Tan, N. S., et al. (2017). Fabrication and Characterization of a Novel Crosslinked Human KeratinAlginate Sponge. J. Tissue Eng. Regen. Med. 11, 2590-2602. doi:10.1002/term.2159

Hassan, C. M., and Peppas, N. A. (2000). Structure and Morphology of Freeze/ Thawed PVA Hydrogels. Macromolecules 33, 2472-2479. doi:10.1021/ ma9907587

Henderson, T. M. A., Ladewig, K., Haylock, D. N., McLean, K. M., and O’Connor, A. J. (2013). Cryogels for Biomedical Applications. J. Mater. Chem. B 1, 2682-2695. doi:10.1039/c3tb20280a

Kim, S. Y., Park, B. J., Lee, Y., Park, N. J., Park, K. M., Hwang, Y.-S., et al. (2019). Human Hair Keratin-Based Hydrogels as Dynamic Matrices for Facilitating Wound Healing. J. Ind. Eng. Chem. 73, 142-151. doi:10.1016/j.jiec.2019.01.017

Konop, M., Czuwara, J., Kłodzińska, E., Laskowska, A. K., Sulejczak, D., Damps, T., et al. (2020). Evaluation of Keratin Biomaterial Containing Silver Nanoparticles as a Potential Wound Dressing in Full-thickness Skin Wound Model in Diabetic Mice. J. Tissue Eng. Regen. Med. 14, 334-346. doi:10.1002/term.2998

Loh, Q. L., and Choong, C. (2013). Three-dimensional Scaffolds for Tissue Engineering Applications: Role of Porosity and Pore Size. Tissue Eng. B: Rev. 19, 485-502. doi:10.1089/ten.TEB.2012.0437

Lozinsky, V. I. (2002). Cryogels on the Basis of Natural and Synthetic Polymers: Preparation, Properties and Application. Russ. Chem. Rev. 71, 489-511. doi:10.1070/RC2002v071n06ABEH000720

Nakata, R., Osumi, Y., Miyagawa, S., Tachibana, A., and Tanabe, T. (2015). Preparation of Keratin and Chemically Modified Keratin Hydrogels and Their Evaluation as Cell Substrate with Drug Releasing Ability. J. Biosci. Bioeng. 120, 111-116. doi:10.1016/j.jbiosc.2014.12.005

Placone, J. K., Navarro, J., Laslo, G. W., Lerman, M. J., Gabard, A. R., Herendeen, G. J., et al. (2017). Development and Characterization of a 3D Printed, Keratin-Based Hydrogel. Ann. Biomed. Eng. 45, 237-248. doi:10.1007/s10439-016-1621-7

Ross, J. B., Szabo, A. G., and Hogue, C. W. (1997). Enhancement of Protein Spectra with Tryptophan Analogs: Fluorescence Spectroscopy of Protein-Protein and Protein-Nucleic Acid Interactions. Methods Enzymol. 278, 151-190. doi:10.1016/s0076-6879(97)78010-8

Sadeghi, S., Nourmohammadi, J., Ghaee, A., and Soleimani, N. (2020). Carboxymethyl Cellulose-Human Hair Keratin Hydrogel with Controlled Clindamycin Release as Antibacterial Wound Dressing. Int. J. Biol. Macromolecules 147, 1239-1247. doi:10.1016/j.ijbiomac.2019.09.251

Saul, J. M., Ellenburg, M. D., de Guzman, R. C., and Dyke, M. V. (2011). Keratin Hydrogels Support the Sustained Release of Bioactive Ciprofloxacin. J. Biomed. Mater. Res. 98A, 544-553. doi:10.1002/jbm.a.33147

Shavandi, A., Silva, T. H., Bekhit, A. A., and Bekhit, A. E.-D. A. (2017). Keratin: Dissolution, Extraction and Biomedical Application. Biomater. Sci. 5, 1699-1735. doi:10.1039/c7bm00411g
Sun, K., Guo, J., He, Y., Song, P., Xiong, Y., and Wang, R.-M. (2016). Fabrication of Dual-Sensitive Keratin-Based Polymer Hydrogels and Their Controllable Release Behaviors. J. Biomater. Sci. Polym. Edition 27, 1926-1940. doi:10.1080/09205063.2016.1239955

Sun, Z., Chen, X., Ma, X., Cui, X., Yi, Z., and Li, X. (2018). Cellulose/keratincatechin Nanocomposite Hydrogel for Wound Hemostasis. J. Mater. Chem. B 6, 6133-6141. doi:10.1039/c8tb01109e

Tang, A., Li, Y., Yao, Y., Yang, X., Cao, Z., Nie, H., et al. (2021). Injectable Keratin Hydrogels as Hemostatic and Wound Dressing Materials. Biomater. Sci. 9, 4169-4177. doi:10.1039/D1BM00135C

Wang, J., Hao, S., Luo, T., Cheng, Z., Li, W., Gao, F., et al. (2017). Feather Keratin Hydrogel for Wound Repair: Preparation, Healing Effect and Biocompatibility Evaluation. Colloids Surf. B: Biointerfaces 149, 341-350. doi:10.1016/ j.colsurfb.2016.10.038

Wang, S., Taraballi, F., Tan, L. P., and Ng, K. W. (2012). Human Keratin Hydrogels Support Fibroblast Attachment and Proliferation In Vitro. Cell. Tissue Res. 347, 795-802. doi:10.1007/s00441-011-1295-2

Wang, S., Wang, Z., Foo, S. E. M., Tan, N. S., Yuan, Y., Lin, W., et al. (2015). Culturing Fibroblasts in 3D Human Hair Keratin Hydrogels. ACS Appl. Mater. Inter. 7, 5187-5198. doi:10.1021/acsami.5b00854

Wang, X., Shi, Z., Zhao, Q., and Yun, Y. (2021). Study on the Structure and Properties of Biofunctional Keratin from Rabbit Hair. Materials 14, 379. doi:10.3390/ma14020379

Yu, D. W. (2002). Raman Spectroscopic Analysis of the Spatial Structure and Conformation of Peptides and Proteins. Chin. J. Anal. Lab. 21, 257-261. doi:10.1002/jrs.1403

Zhai, M., Xu, Y., Zhou, B., and Jing, W. (2018). Keratin-chitosan/n-ZnO Nanocomposite Hydrogel for Antimicrobial Treatment of Burn Wound Healing: Characterization and Biomedical Application. J. Photochem. Photobiol. B: Biol. 180, 253-258. doi:10.1016/j.jphotobiol.2018.02.018

Zhang, Y., Zhao, W., and Yang, R. (2015). Steam Flash Explosion Assisted Dissolution of Keratin from Feathers. ACS Sustain. Chem. Eng. 3, 2036-2042. doi:10.1021/acssuschemeng.5b00310

Zhao, Z., Moay, Z. K., Lai, H. Y., Goh, B. H. R., Chua, H. M., Setyawati, M. I., et al. (2020). Characterization of Anisotropic Human Hair Keratin Scaffolds Fabricated via Directed Ice Templating. Macromol. Biosci. 21, 2000314, doi:10.1002/mabi.202000314

Conflict of Interest: The authors declare that they have no known competing financial interests or personal relationships that could have appeared to influence the work reported in this paper.

Publisher's Note: All claims expressed in this article are solely those of the authors and do not necessarily represent those of their affiliated organizations, or those of the publisher, the editors, and the reviewers. Any product that may be evaluated in this article, or claim that may be made by its manufacturer, is not guaranteed or endorsed by the publisher.

Copyright (c) 2021 Wang, Shi, Zhao and Shen. This is an open-access article distributed under the terms of the Creative Commons Attribution License (CC $B Y$ ). The use, distribution or reproduction in other forums is permitted, provided the original author(s) and the copyright owner(s) are credited and that the original publication in this journal is cited, in accordance with accepted academic practice. No use, distribution or reproduction is permitted which does not comply with these terms. 